\title{
Performance Evaluation of a Modified SweepSAR Mode for Quad-Pol Application in SAR Systems
}

\author{
Jung-Hwan $\operatorname{Lim}^{1} \cdot$ Jae Wook Lee ${ }^{1, *} \cdot$ Taek-Kyung Lee ${ }^{1} \cdot$ Hyeon-Cheol Lee ${ }^{2}$. \\ Sang-Gyu Lee ${ }^{2}$ Sang-Burm Ryu ${ }^{2} \cdot$ Seong-Sik Yoon ${ }^{3}$
}

\begin{abstract}
Efficient earth observation using spaceborne synthetic aperture radar (SAR) requires the analysis of high-resolution wide-swath (HRWS) systems, which have a fine resolution and a short revisit time through wide-area observation, and quadrature-polarimetric (quad-pol) systems, which can obtain a quad-pol image and have many applications. The operational method of SAR generally affects system performance involving the system parameters and antenna patterns, and thus analyzing HRWS quad-pol SAR systems is essential to the system design. In this paper, an extension of the modified SweepSAR technique to a quad-pol observation scenario is performed and a comparison to ScanSAR and conventional SweepSAR is provided for a simulated scenario. On the basis of the system requirements, we selected the optimal parameters and designed a reflector antenna suitable for a wide-swath quad-pol SAR system. As a result of comparing the wide-swath quad-pol SAR operation modes, the modified SweepSAR mode demonstrated advantages in terms of system performance, such as the ambiguity ratio and swath width, and reflector antenna design for a spaceborne SAR system.
\end{abstract}

Key Words: High-Resolution Wide-Swath (HRWS), Quadrature-Polarimetric (Quad-Pol), Reflector Antenna, Synthetic Aperture Radar (SAR).

\section{INTRODUCTION}

A spaceborne synthetic aperture radar (SAR) is a sensor that is mounted on a satellite and utilizes radio waves to enable highresolution and all-weather Earth observation. However, conventional spaceborne SAR has some limitations in use because it has a trade-off relationship within the main system parameters. In addition, it obtains information from the Earth's surface by transmitting and receiving only a single polarization.

In Earth observation using spaceborne SAR, an efficient observation requires a short revisit time by observing wide areas [1].
For this purpose, wide-swath SAR operation modes, such as ScanSAR and TOPSAR (terrain observation with progressive scans SAR), were introduced and studied [2, 3]. However, these two modes observe the sub-swaths sequentially and this operational method worsens the resolution in the azimuthal direction of the SAR system. Therefore, obtaining a wide-swath and fine resolution at the same time is difficult for a conventional wideswath SAR system. To overcome this constraint, many operation modes are currently being studied as high-resolution wideswath (HRWS) systems. Among these systems, we are interested in HRWS systems using a reflector antenna [4-6]. Generally,

Manuscript received March 13, 2020 ; Revised May 25, 2020 ; Accepted June 8, 2020. (ID No. 20200313-034J)

${ }^{1}$ Department of Electronics and Information Engineering, Korea Aerospace University, Goyang, Korea.

${ }^{2}$ Satellite Payload Development Division, Korea Aerospace Research Institute, Daejeon, Korea.

${ }^{3}$ Imaging Radar Team, Hanwha Systems, Yongin, Korea.

"Corresponding Author: Jae Wook Lee (e-mail: jwlee1@kau.ac.kr)

This is an Open-Access article distributed under the terms of the Creative Commons Attribution Non-Commercial License (http://creativecommons.org/licenses/by-nc/4.0) which permits unrestricted non-commercial use, distribution, and reproduction in any medium, provided the original work is properly cited.

(c) Copyright The Korean Institute of Electromagnetic Engineering and Science. All Rights Reserved. 
reflector antennas have high gain and moderate bandwidth and can be made light-weight by using composite material or with a mesh-type design. In addition, digital beam forming (DBF) can be performed by on/off operation of the feed elements. One of the operation modes analyzed as a reflector-based HRWS system is SweepSAR [4], which is a SAR operation mode of obtaining a high-resolution image using a broad beam to transmit and a narrow beam to receive based on the SCORE(scan-onreceive) method using DBF. However, this operation mode requires the use of a low pulse repetition frequency (PRF) to obtain an unambiguous wide swath. For this reason, a modified mode of SweepSAR using dual channels, which is called MSweepSAR, was examined for a single polarization (single-pol) SAR system [6].

Generally, the SAR system employs only a single transmitting/receiving polarization and obtains one type of polarization information. In contrast, in polarimetric SAR, such as dualpolarimetric and quadrature-polarimetric (quad-pol) approaches, various information can be obtained using multiple transmitting and receiving polarizations, and it has been utilized this way in many fields $[7,8]$. However, for a SAR system operating in a quad-pol mode, interleaving transmitting pulses of different polarizations impacts the design of the SAR system. Therefore, accurately analyzing this effect, as done in $[9,10]$, is necessary to design an appropriate quad-pol SAR system.

In the current paper, the reflector antenna and the performance of the proposed quad-pol system operating in MSweepSAR mode are designed and analyzed, respectively. We also compared wide-swath quad-pol SAR systems in terms of system performance and antenna design.

\section{PERFORMANCE OF THE WIDE-SWATH}

\section{QUAD-POL SAR SYSTEM}

Generally, a SAR system can be characterized by performance indicators, such as the ambiguity-to-signal ratio and resolution. These indicators are strongly related to the system $\mathrm{pa}^{-}$ rameters, antenna patterns, and SAR operation modes.

The ambiguity-to-signal ratio, which comprises the range ambiguity-to-signal ratio (RASR) and azimuth ambiguity-tosignal ratio (AASR), is the ratio of undesired signals to the desired SAR signal. it is calculated through the transmitting/receiving antenna pattern, $G_{t}(\theta) / G_{r}(\theta)$; backscattering coefficient, $\sigma^{0}$; slant range, $\rho$; range ambiguous slant range, $\rho_{R A}$; incidence angle, $\theta_{I}$; Doppler processing bandwidth, $P B$; and the angles at which the ambiguity signal occurs in the range and azimuth directions, $\theta_{A}$ and $\theta_{A Z}$, respectively $[9,11]$. The resolution, which is the degree of distinguishing the main target from other objects, in the azimuthal direction $\left(\rho_{a}\right)$, is calculated through the beam footprint velocity $\left(V_{g}\right)$, Doppler bandwidth $\left(\Delta f_{\text {dop }}\right)$ and wavelength $(\lambda)[12,13]$. The range resolution is related to the bandwidth of the transmitted pulse and is not directly associated with other performance indicators.

$$
\begin{gathered}
R A S R=\frac{\sum_{n \neq 0} \int_{P B} \frac{\sigma^{0}\left(\theta_{I}(n)\right) G_{t}\left(\theta_{A}(n), \theta_{A Z}\right) G_{r}\left(\theta_{A}(n), \theta_{A Z}\right)}{\rho_{R A}^{3}(n) \sin \left(\theta_{I}(n)\right)} d \theta_{A Z}}{\int_{P B} \frac{\sigma^{0}\left(\theta_{I}(0)\right) G_{t}\left(\theta_{A}(0), \theta_{A Z}\right) G_{r}\left(\theta_{A}(0), \theta_{A Z}\right)}{\rho^{3} \sin \left(\theta_{I}(0)\right)} d \theta_{A Z}} \\
A A S R=\frac{\sum_{n \neq 0} \int_{P B} G_{t}\left(\theta_{A Z}(n)\right) G_{r}\left(\theta_{A Z}(n)\right) d \theta_{A Z}(n)}{\int_{P B} G_{t}\left(\theta_{A Z}(0)\right) G_{r}\left(\theta_{A Z}(0)\right) d \theta_{A Z}(0)} \\
\rho_{a}=\frac{0.886 V_{g}}{\Delta f_{d o p}}=\frac{0.443 \lambda}{P B}
\end{gathered}
$$

We dealt with the wide-swath and quad-pol operation modes and designed the antenna, which is one of the most important parts of the SAR system, to conduct a proper performance analysis.

\section{Wide-Swath SAR System}

The swath width of the spaceborne SAR system is affected by the SAR system parameters, such as the PRF, pulse width, altitude of the satellite, and incidence angle. Moreover, the swath width is also related to noise and the resolution performance. As a representative operation mode, ScanSAR, the most widely used wide-swath SAR operation mode, uses multiple beams to sequentially observe sub-swaths, which are designed according to the position of the blind ranges determined by the PRF and pulse width.

However, as the sub-swaths are observed sequentially, the dwell time of each sub-swath is decreased, and the azimuth resolution is degraded in proportion to the number of sub-swaths [2]. In contrast, SweepSAR continuously observes the total swath width by using a broad transmitting antenna beam and narrow receiving antenna beams through a DBF method, whereas ScanSAR uses the same transmitting/receiving pattern for each sub-swath. However, due to the azimuth ambiguity performance and blind range, conventional SweepSAR requires a low PRF and a large reflector antenna.

Alternatively, M-SweepSAR, which is examined in this paper, can overcome this constraint by using two channels, so that this operation mode can reduce the reflector size than SweepSAR. M-SweepSAR uses two rows of linear-array feed antennas, with each of the two channels corresponding to a row. Each channel forms a transmitting/receiving pattern in the same way as the DBF method of SweepSAR. Unlike SweepSAR, however, an area which overlaps the blind range of one channel can be observed by using the other channel. In other 
words, each channel operates simultaneously with different PRFs. Two channels continuously observe the total swath with one of the two frequency bands within the total available bandwidth and filtering is performed to reduce interference $[6,14]$. The operational concepts of the three wide-swath modes (ScanSAR, SweepSAR, and M-SweepSAR) are shown in Fig. 1.

In this way, the required antenna conditions depend on the SAR operation mode. The selection of the SAR system parameters, such as the PRF, is also affected in meeting the system requirements.

\section{Quad-Pol SAR System}

In the quad-pol SAR operation, $\mathrm{H} / \mathrm{V}$ polarization is transmitted and received alternately within the given intervals, and the PRF is doubled that of the single-pol SAR. Therefore, the quad-pol SAR can obtain four types of information: $\mathrm{HH}, \mathrm{HV}$, $\mathrm{VH}$, and VV. However, the increase in the PRF causes a decrease of the receiving interval and performance degradation in the RASR.

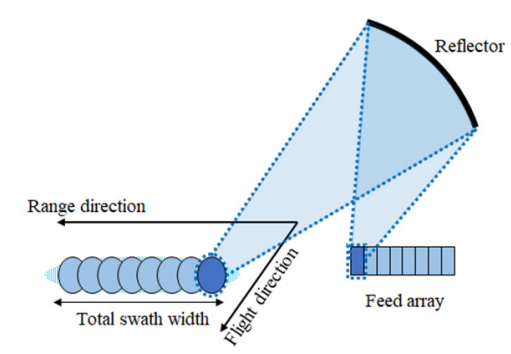

(a)

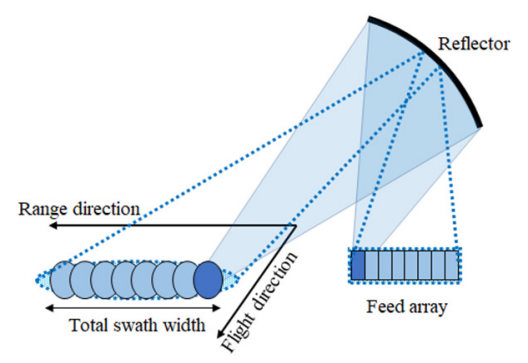

(b)

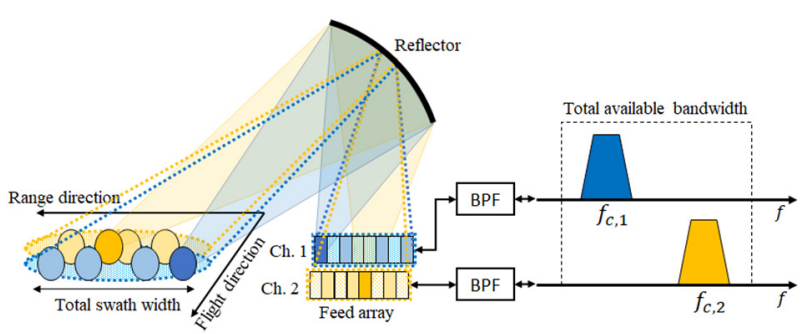

(c)

Fig. 1. Operational concepts of the wide-swath SAR systems: (a) ScanSAR, (b) SweepSAR, and (c) M-SweepSAR. Dashed line is transmitting operation; colored region is receiving operation. Adapted from Lim [13].
Moreover, the behavior of backscattering coefficients in different polarizations $[15,16]$ needs to be considered for the prediction of the quad-pol SAR performance. Due to the interleaving of the transmitting pulses of different polarizations, two types of signals with different transmitting polarization channels but the same receiving polarization channels are considered as range ambiguities. Since cross-polarized backscatter levels are typically lower than the co-pol ones and the first-order ambiguities, in Eq. (1), dominate the range ambiguity levels when considering the antenna radiation pattern, poorer RASR performance is expected for cross-pol in a conventional quad-pol SAR system [9]. For these reasons, when we assume the same effective channel PRF, the quad-pol mode obtains about half the swath width of the single-pol mode.

Therefore, the considerable effects of the use of the quad-pol mode on the SAR system performance need to be analyzed. In addition, performance degradation should be compensated with the selection of appropriate parameters based on the relation between the SAR system parameters and the system performance $[17,18]$. The increase in the PRF leads to the degradation of the RASR performance while improving the AASR performance. $\mathrm{PB}$, which is the reliable range in the azimuthal direction, also affects ambiguity performances. It is especially important in the AASR characteristics, which are related to the azimuth resolution, rather than to the RASR.

\section{Design of the SAR Reflector Antenna}

For satisfying the required performance of a SAR system, a proper antenna should be considered and designed depending on the polarization. In other words, it is necessary to design an antenna that considers quad-pol performance because there is a limit to achieving sufficient quad-pol performance by appropriately selecting the system parameters while using an antenna that had been considered for only single mode in its design [18]. According to a brief comparison between two systems employing the single-pol mode and the quad-pol mode, the ambiguity performance of the single-pol mode is better than that of the quad-pol mode. In terms of resolution, the characteristic of the quad-pol mode is better than that of the single-pol because ScanSAR, which has a trade-off relation between resolution and the number of sub-swaths, covers less swath width due to the increase of the PRF and the RASR performance degradation, especially at the far region, in the quad-pol mode. Therefore, the antenna should be designed to match the ambiguity ratio of the quad-pol mode and the resolution of the single-pol mode.

The relation among the size of the reflector antenna, ambiguity ratio, and resolution of the SAR system is as follows. Increasing the reflector length in the azimuthal direction improves the azimuth ambiguity performance and degrades the resolution regardless of the operation mode. Increasing the reflector width 
in the range direction improves the range ambiguity performance in ScanSAR. However, as the beamwidth decreases, the space between the feeds becomes narrower considering the beam deviation factor (BDF) to ensure a continuous total swath width, and the resolution performance is degraded because it leads to an increased number of sub-swaths. The $B D F$ of an offset reflector antenna is derived from the beam scan angle, $\theta_{B}$; feed tilt angle, $\theta_{F}$; focal length, $F$; and parent reflector, $D_{P}$; as shown in the following [19]:

$$
B D F=\frac{\theta_{B}}{\theta_{F}}=\frac{1+0.36\left(4 \frac{F}{D_{p}}\right)^{-2}}{1+\left(4 \frac{F}{D_{p}}\right)^{-2}}
$$

As SweepSAR and M-SweepSAR continuously observe the full range of the swath width, improving the range ambiguity ratio by increasing the reflector width does not affect the resolution. However, as the reflector width increases, the space between the feeds becomes narrower and the minimum space is limited by the size of the feed antenna. Therefore, RASR and AASR require an increase in the reflector size, but the resolution and the size of the feed antenna must be considered as the upper bound of the reflector width $[13,20]$.

\section{CHARACTERISTIC ANALYSIS AND COMPARISON OF}

\section{THE QUAD-POL M-SWEEPSAR SYSTEM}

In this research, we selected a spaceborne SAR system with a $\mathrm{C}$-band center frequency operating at an altitude of $505 \mathrm{~km}$ with a total swath width of $150 \mathrm{~km}$ for a single-pol mode at an incidence angle of $20^{\circ}-35^{\circ}$ [6]. In this case, we assumed that the SAR system performance, which involves an ambiguity ratio in each direction and the resolution should be under the conditions of $-20 \mathrm{~dB}$ and $10 \mathrm{~m}$, respectively.

The required SAR antenna beamwidth was determined by the swath width and the number of sub-swaths, which help in selecting the optimum reflector antenna depending on the operation mode. This paper suggests a reflector length for the offset reflector antennas of $4 \mathrm{~m}, 13.5 \mathrm{~m}$, and $6 \mathrm{~m}$ for ScanSAR, SweepSAR, and M-SweepSAR, respectively. These antennas had a reflector with $F / D_{p}=0.409$ and a feed array linearly placed in the range direction with one row. Only M-SweepSAR had a feed array with two rows. The radiation patterns of the reflector antenna by several elements at one row of the feed array for the M-SweepSAR system are presented in Fig. 2. These patterns were simulated using the FEKO EM simulation tool. The transmitting pattern in the elevation direction was broad enough to cover the overall swath using additional feed antennas at both ends of each row of the feed array. The receiv-

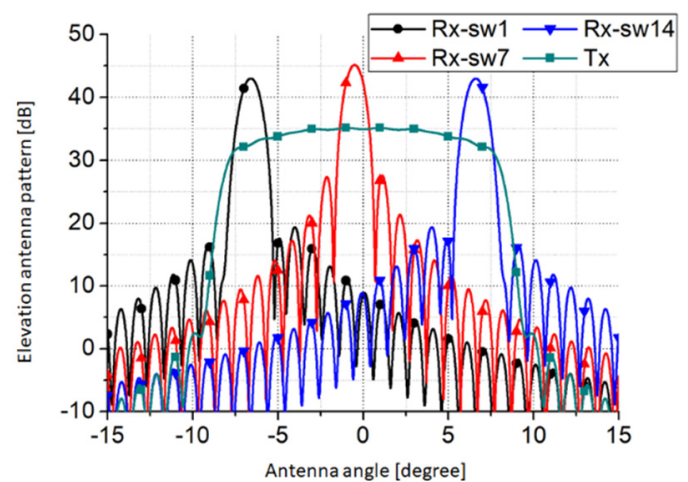

(a)

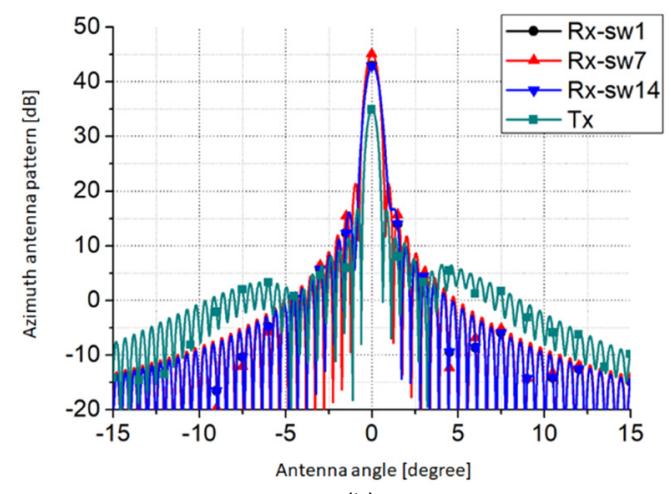

(b)

Fig. 2. Antenna radiation patterns used for the quad-pol MSweepSAR system: (a) elevation patterns and (b) azimuth patterns.

ing patterns were tilted in the elevation direction, so that each feed received echoes from each sub-swath.

The reflector antenna of each SAR system had a feed antenna according to the number of sub-swaths in the single-pol mode. However, SAR systems use fewer feed antennas in the quad-pol mode to meet the requirements (except for the MSweepSAR system). In quad-pol M-SweepSAR, the PRF and signal acquisition areas of each channel were determined based on the timing diagram shown in Fig. 3. M-SweepSAR had 14

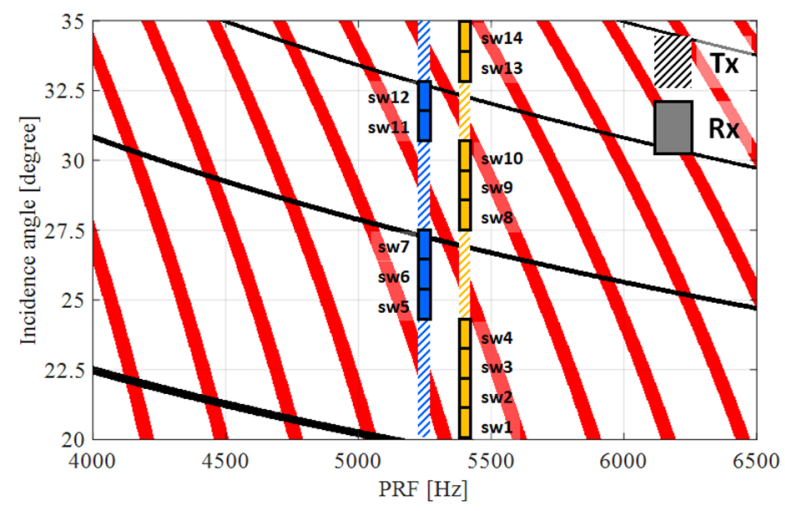

Fig. 3. Timing diagram of the quad-pol M-SweepSAR system. Red lines are blind range; black lines, nadir return; dashed region, channel PRF selection; colored segments, subswaths corresponding to each feed element. 
sub-swaths in the quad-pol mode with PRFs of $5,250 \mathrm{~Hz}$ and $5,400 \mathrm{~Hz}$ in each row of the feed array, which maintained an PB of $0.16^{\circ}$ and had 14 sub-swaths in a single-pol mode. As shown in Fig. 3, each row of the feed array transmits the signal to sub-swath (sw) 1-14, with one row receiving echoes from sw 5-7 and 11-12, and the other row receiving echoes from sw 1-4, 8-10, and 13-14. ScanSAR had three sub-swaths in the quadpol mode, with three PRFs of $10.15,10$, and $9 \mathrm{kHz}$ and a PB of $0.44^{\circ}$, and 6 sub-swaths in the single-pol mode. SweepSAR had 13 sub-swaths in the quad-pol mode, with a PRF of 3,400 $\mathrm{Hz}$ and $\mathrm{PB}$ of $0.26^{\circ}$, and 24 sub-swaths in the single-pol mode. All of the information for the three wide-swath operation modes working in the quad-pol mode is shown in Table 1.

Based on the simulation results of the three quad-pol SAR systems for wide-swath, the SAR performances, including reflector antenna size, ambiguity ratio, resolution, and swath width, were analyzed and compared with the use of the backscattering coefficients assumption, which was suggested by Ulaby and Dobson [16]. In our previous work [18], we had conducted a comparison between our calculation results and data in [9] to verify our analysis procedure on quad-pol SAR systems. Generally, the RASR for the co-pol (HH and VV) was about $10 \mathrm{~dB}$ lower than that for the cross-pol ( $\mathrm{HV}$ and $\mathrm{VH})$. Therefore, we present only the RASR for HV in Fig. 4.

From Table 1 and Fig. 4, the reflector length for SweepSAR was the largest in order to meet the required performance in the single-pol and quad-pol modes while maintaining the required swath width obtained in the single-pol mode because of the

Table 1. Comparison of the wide-swath quad-pol SAR operation modes

\begin{tabular}{lccc}
\hline \multicolumn{1}{c}{ Operation mode } & ScanSAR & SweepSAR & M-SweepSAR \\
\hline Reflector length (m) & 4 & 13.5 & 6 \\
Number of feeds & 6 & 26 & 32 \\
& & $($ Tx: 26, & $($ Tx: $16 \times 2$, \\
& & Rx: 24$)$ & Rx: $14 \times 2)$ \\
Number of sub-swaths & 3 & 13 & 14 \\
Swath width (km) & 70 & 75 & 150 \\
(incidence angle, $\left.{ }^{\circ}\right)$ & $(20-27.5)$ & $(20-28.1)$ & $(20-35)$ \\
PRF (Hz) & 10,150 & 3,400 & $5,250(\mathrm{Ch} .1)$ \\
& 10,000 & & $5,400(\mathrm{Ch} .2)$, \\
& 9,000 & & 0.16 \\
PB (o) & 0.44 & 0.26 & 8.9 \\
Azimuth resolution & 9.7 & 5.4 & \\
(m) & & & \\
RASR/AASR (dB) & & $<-20$ & \\
\hline
\end{tabular}

characteristics of the SweepSAR operation mode. Conversely, the reflector lengths of M-SweepSAR and ScanSAR were half that of SweepSAR and the smallest, respectively. Quad-pol SweepSAR had the largest reflector antenna but was best in terms of total ambiguity ratio, which means the sum of RASR and AASR. Quad-pol ScanSAR and quad-pol M-SweepSAR had similar levels in ambiguity within the incidence angle range of $20.0^{\circ}-27.5^{\circ}$. However, it can be conjectured from Fig. 4 that quad-pol M-SweepSAR had better characteristics than quadpol ScanSAR and satisfied the requirements on the overall incidence angle range.

The azimuth resolution depends on the Doppler bandwidth, and in the case of ScanSAR, it is also affected by the number of sub-swaths. As shown by the simulation results, quad-pol ScanSAR had the largest PB with the smallest antenna size, but its resolution was the worst. In addition, quad-pol SweepSAR had the best resolution among the three operation modes, even though it had the largest antenna size because quad-pol M-SweepSAR had a narrower PB for the azimuth ambiguity performance.

In terms of the swath width under the conditions of less than $-20 \mathrm{~dB}$ RASR and AASR, quad-pol ScanSAR was limited in

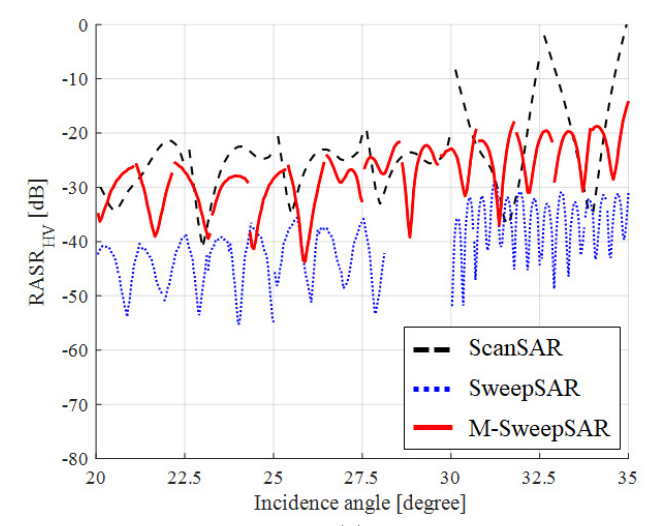

(a)

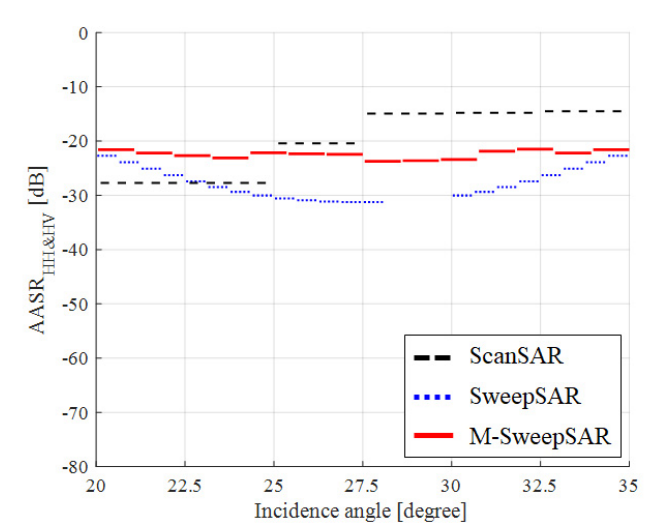

(b)

Fig. 4. Comparison of the ambiguity performance of the quad-pol SAR system according to the operation mode: (a) RASR and (b) AASR. 
obtaining a wide swath width because of the ambiguity performance degradation over a large incidence angle range. As the $\mathrm{PRF}$ increased, and the receiving interval was reduced for the quad-pol mode, quad-pol SweepSAR was also limited in obtaining a continuous total swath width because of the blind range. Therefore, the two modes had half of the total swath width in comparison with the single-pol mode. On the contrary, quad-pol M-SweepSAR could obtain a swath width almost like that of the single-pol mode within the required performance levels. In other words, M-SweepSAR used two PRFs, and this characteristic was advantageous in obtaining a wider swath width than SweepSAR. In addition, M-SweepSAR had a better ambiguity performance than ScanSAR; it was also advantageous in obtaining a wider swath width than ScanSAR.

Most of the differences in the performance indicators discussed in this chapter arose from the differences in antenna patterns, which were determined on the basis of the required performance. Therefore, if the requirements change, the antenna size, which determines the achievable range of the SAR system performance, should be changed. As a result of confirming the performance changes derived from the size of the reflector antenna in each operation mode, increasing the reflector size for the ScanSAR improves the ambiguity performance but worsens the resolution [20]. Conversely, SweepSAR and M-SweepSAR have the advantage of maintaining resolution in comparison with ScanSAR even when the reflector size increases, and MSweepSAR can reduce the reflector size in comparison with SweepSAR, as mentioned in Chapter II. In other words, for the ScanSAR system to obtain a wider swath width in the quad-pol, a larger reflector must be required to improve the ambiguity ratios, which does not satisfy the resolution requirement. However, we can design a reflector antenna for the M-SweepSAR and achieve a wider swath width in the quad-pol, although it uses a larger reflector than ScanSAR.

\section{CONCLUSION}

In this paper, the performance analysis of the proposed quadpol M-SweepSAR system was conducted. To determine the validity of our analysis, we designed, analyzed, and compared the ScanSAR, SweepSAR, and M-SweepSAR systems. The result of the analysis and comparison of the three operation modes based on the properly designed systems indicated that M-SweepSAR is expected to be highly recommended as the HRWS spaceborne SAR system because it is advantageous in satisfying the required ambiguity ratio and resolution in both the single-pol and the quad-pol modes with an appropriate reflector antenna size and obtains a wider swath width in the quad-pol mode than the conventional wide-swath operation mode. However, as the feed array has two rows, the system must have dual channels and operate two center frequencies in the available bandwidth. Therefore, filtering for interference suppression should be considered for practical implementation.

This work was performed for the Development of Radar Payload Technologies for Compact Satellite in Korea Aerospace Research Institute, funded by the Ministry of Science and ICT.

\section{REFERENCES}

[1] A. Currie and M. A. Brown, "Wide-swath SAR," IEE Proceedings $F$ (Radar and Signal Processing), vol. 139, no. 2, pp. 122-135, 1992.

[2] D. D'Aria, and F. D. Zan, D. Giudici, A. M. Guarnieri, and F. Rocca, "Burst-mode SARs for wide-swath surveys," Canadian Journal of Remote Sensing, vol. 33, no. 1, pp. 27-38, 2007.

[3] F. De Zan and A. M. Guarnieri, "TOPSAR: terrain observation by progressive scans," IEEE Transactions on Geoscience and Remote Sensing, vol. 44, no. 9, pp. 2352-2360, 2006.

[4] A. Freeman, G. Krieger, P. Rosen, M. Younis, W. T. Johnson, S. Huber, R. Jordan, and A. Moreira, "SweepSAR: beam-forming on receive using a reflector-phased array feed combination for spaceborne SAR," in Proceedings of 2009 IEEE Radar Conference, Pasadena, CA, 2009, pp. 1-9.

[5] M. Villano, G. Krieger, and A. Moreira, "Staggered SAR: high-resolution wide-swath imaging by continuous PRI variation," IEEE Transactions on Geoscience and Remote Sensing, vol. 52, no. 7, pp. 4462-4479, 2014.

[6] S. S. Yoon, J. W. Lee, T. K. Lee, S. B. Ryu, H. C. Lee, and S. G. Lee, "A modified SweepSAR mode with dual channels for high resolution and wide swath," Journal of Electromagnetic Engineering and Science, vol. 18, no. 3, pp. 199-205, 2018.

[7] V. Turkar, R. Deo, S. Hariharan, and Y. S. Rao, "Comparison of classification accuracy between fully polarimetric and dual-polarization SAR images," in Proceedings of 2011 IEEE International Geoscience and Remote Sensing Symposium, Vancouver, Canada, 2011, pp. 440-443.

[8] Y. Yamaguchi, S. Sengoku, H. Yamada, and R. Sato, "Scattering power decomposition results of ALOS2 quad pol data along Amazon River," in Proceedings of the 12th European Conference on Synthetic Aperture Radar (EUSAR), Aachen, Germany, 2018, pp. 1-4.

[9] M. Villano, G. Krieger, and A. Moreira, "New insights into ambiguities in quad-pol SAR," IEEE Transactions on Geoscience and Remote Sensing, vol. 55, no. 6, pp. 3287-3308, 2017. 
[10] R. K. Raney, A. Freeman, and R. L. Jordan, "Improved range ambiguity performance in quad-pol SAR," IEEE Transactions on Geoscience and Remote Sensing, vol. 50, no. 2, pp. 349-356, 2012.

[11] W. A. Imbriale, Spaceborne Antennas for Planetary Exploration. New York, NY: John Wiley \& Sons, 2006, pp. 305317.

[12] I. G. Cumming and F. H. Wong, Digital Processing of Synthetic Aperture Radar Data: Algorithms and Implementation. Boston, MA: Artech House, 2005, pp. 113-157.

[13] J. H. Lim, "A study on process implementation for performance analysis of C-band quad-pol SAR systems," M.S. thesis, Department of Electronics and Information Engineering, Korea Aerospace University, Goyang, Korea, 2020.

[14] F. Bordoni, G. Krieger, and M. Younis, "Multifrequency subpulse SAR: exploiting chirp bandwidth for an increased coverage," IEEE Geoscience and Remote Sensing Letters, vol. 16, no. 1, pp. 40-44, 2018.

[15] T. Jin and Y. Oh, "An improved semi-empirical model for radar backscattering from rough sea surfaces at Xband," Journal of Electromagnetic Engineering and Science,

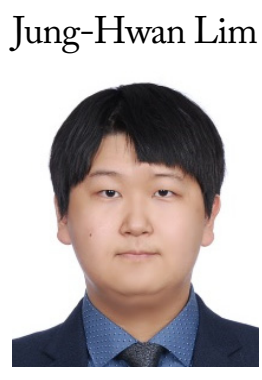

received his B.S. and M.S. degrees in electronics and information engineering from Korea Aerospace University (KAU), Goyang, Korea, in 2018 and 2020, respectively. He is currently working toward a Ph.D. degree at the Microwave and Millimeterwave Solution Laboratory of KAU. His current research interests include satellite communications/radar antennas, spaceborne SAR systems, and EMI/EMC.

Jae Wook Lee

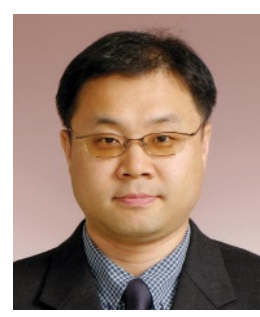

received his B.S. degree in electronic engineering from Hanyang University, Seoul, Korea, in 1992, and his M.S. and Ph.D. degrees in electrical engineering with a specialization in electromagnetics from the Korea Advanced Institute of Science and Technology, Daejeon, Korea, in 1994 and 1998, respectively. From 1998 to 2004, he was a senior member of the Advanced Radio Technology Department, Radio and Broadcasting Research Laboratory, Electronics and Telecommunications Research Institute, Daejeon. He was with the faculty of the Korea Aerospace University (KAU), Goyang, Korea. He is currently a professor at the School of Electronics and Information Engineering of KAU. His current research interests include high-power amplifier design, computational electromagnetics, electromagnetic interference/electro-magnetic compatibility analysis on printed circuit boards, satellite antennas, and spaceborne SAR systems. vol. 18, no. 2, pp. 136-140, 2018.

[16] F. T. Ulaby and M. C. Dobson, Handbook of Radar Scattering Statistics for Terrain. Norwood, MA: Artech House, 1989.

[17] H. M. Braun, "A new SAR sensor designed for microsatellites," in Proceedings of 2010 IEEE International Geoscience and Remote Sensing Symposium, Honolulu, HI, 2010, pp. 213-215.

[18] J. H. Lim, S. S. Yoon, J. W. Lee, T. K. Lee, S. B. Ryu, H. C. Lee, and S. G. Lee, "Performance analysis of quad-pol SAR system for wide-swath operation mode," The Journal of Korean Institute of Electromagnetic Engineering and Science, vol. 30, no. 2, pp. 141-151, 2019.

[19] W. L. Stutzman and G. A. Thiele, Antenna Theory and Design, 3rd ed. New York, NY: John Wiley \& Sons, 2013, pp. 407-410.

[20] J. H. Lim, J. W. Lee, T. K. Lee, S. B. Ryu, H. C. Lee, and S. G. Lee, "Optimal Design of the Reflector Antenna to Improve Performance of C-Band Quad-Pol ScanSAR Systems," Journal of Electromagnetic Engineering and Science, vol. 20, no. 2, pp. 155-157, 2020.

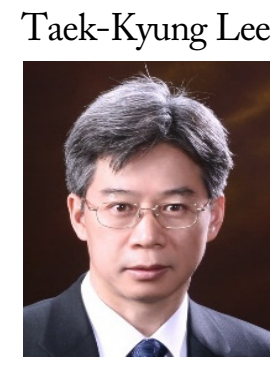

received his B.S. degree in electronic engineering from Korea University, Seoul, Korea, in 1983, and his M.S. and Ph.D. degrees in electrical engineering from the Korea Advanced Institute of Science and Technology (KAIST), Daejeon, Korea, in 1985 and 1990, respectively. From 1990 to 1991, he was a post-doctoral fellow at the University of Texas at Austin, TX, USA, under a grant from the Korea Science and Engineering Foundation. From 1991 to 1992, he was a research scientist at KAIST. In 1992, he joined the faculty of Korea Aerospace University (KAU), Goyang, Korea. He was an associate visiting research professor at the University of Illinois at Urbana-Champaign, IL, USA, from 2001 to 2002. From 2006 to 2007, he was the chairman of the School of Electronics, Information, and Computer Engineering at KAU, where he was the director of the Aerospace and Aviation Electronics Research Center, from 2011 to 2013. He was the Chairman of the Radar Technical Group, Korean Institute of Electromagnetic Engineering and Science, Seoul, from 2012 to 2013, and he served as the president of this institute in 2014. He is currently a professor at the School of Electronics and Information Engineering, KAU. His current research interests include computational electromagnetics, antennas, microwave passive circuits, satellite antennas and spaceborne SAR systems, and air surveillance systems. 


\section{Hyeon-Cheol Lee}

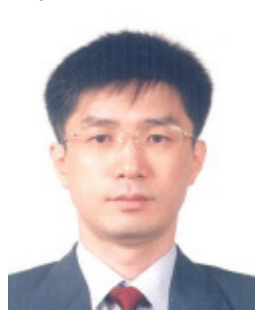

received his B.S. and the M.S. degrees in electronic engineering from Kyungpook National University in 1987 and 1989, respectively, and his Ph.D. degree in electrical engineering from Texas A\&M University in 2001. From 1989 to 1996, he worked at the Agency for Defense Development (ADD) to modify/operate Target Tracking Control Systems for testing Surface-to-Air Missiles and develop the datalink of surveillance UAVs. In 2002, he worked at the Department of Computer Science in Texas A\&M University as a post-doctorate researcher, detecting image contours with an adaptive threshold algorithm. He joined in 2003 at the Korea Aerospace Research Institute (KARI) to develop datalinks and an Auto-tracking System of Vertical Takeoff Landing UAVs. He is recently developing satellite SAR payloads.

\section{Sang-Gyu Lee}

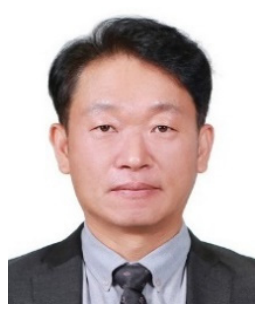

received his B.S., M.S., and Ph.D. degrees in electronic engineering from Chungnam National University in 1993, 1995, and 2011, respectively. During 1993-1995, he focused his research in MPEG2 scalability codec and constant bit rate control mechanisms. Since 1995, he has been in charge of the development of very-high-speed satellite image downlink subsystems of KOMPSATs with the Korea Aerospace Research Institute (KARI), Daejeon, Korea. From 2013 to 2015, he was the program manager of KARI's Internal Research and Development (IRND) program, development of phased array antenna for satellite data transmission. Since 2014, he has been in charge of the development of radar payload technologies for the compact satellite. He was the head of payload electronics team of KARI from 2015.

\section{Sang-Burm Ryu}

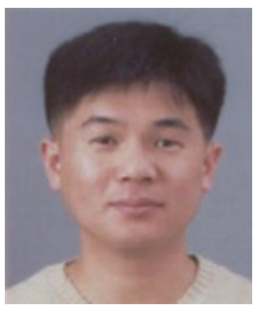

received his B.S. degree in electronic engineering from Daejeon National University of Technology in Daejeon, Korea in 1996 and his M.S. and Ph.D. degrees in the Department of Electronic Engineering from Chungbuk National University, Korea in 2001 and 2010, respectively. Since 2011, he has been working as a senior engineer with the Satellite Payload Development Division, Satellite R\&D Head

Office, Korea Aerospace Research Institute, Daejeon, Korea. His research interests include spaceborne SAR systems, satellite communications, and spaceborne microwave radiometers.

\section{Seong-Sik Yoon}

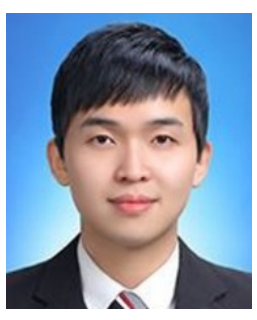

received his B.S., M.S., and Ph.D. degrees in electronic engineering from Korea Aerospace University, Goyang, Korea, in 2010, 2013, and 2018, respectively. He is currently senior researcher at the Satellite $\mathrm{R} \& \mathrm{D}$ center of Hanwha Systems. His current research interests include satellite communication antennas, radar antenna design and analysis, and spaceborne SAR systems. 